\title{
Comparison of DIPSI guidelines versus conventional OGTT for diagnosis of gestational diabetes mellitus
}

\author{
Grishma Girish Desai ${ }^{1 *}$, Pundalik Sonawane ${ }^{2}$
}

\begin{abstract}
${ }^{1}$ Department of Obstetrics and Gynecology, Grant Medical College, Mumbai, Maharashtra, India
${ }^{2}$ Department of Obstetrics and Gynecology, KJ Somaiya Medical College, Mumbai, Maharashtra, India
\end{abstract}

Received: 19 May 2018

Accepted: 26 June 2018

\author{
*Correspondence: \\ Dr. Grishma Girish Desai, \\ E-mail: d.grishma@gmail.com
}

Copyright: (c) the author(s), publisher and licensee Medip Academy. This is an open-access article distributed under the terms of the Creative Commons Attribution Non-Commercial License, which permits unrestricted non-commercial use, distribution, and reproduction in any medium, provided the original work is properly cited.

\begin{abstract}
Background: Gestational Diabetes Mellitus (GDM) is defined as carbohydrate intolerance with recognition or onset during pregnancy is associated with a higher rate of maternal and fetal compromise. OGTT is the current gold standard for screening for GDM. It is a two-step test which requires the pregnant woman to be in a fasting state for a long duration. DIPSI is a one-step procedure for diagnosing GDM does not require patients in a fasting state and is a simple, economical and feasible alternate in Indian scenario. The aim of this study is to compare DIPSI criteria-based test with conventional OGTT for diagnosis of GDM.

Methods: A hospital-based screening study was conducted at Department of Obstetrics and Gynecology, K J Somaiya Medical College and hospital, Mumbai for duration of May 2015 to June 2016. A total of 200 consecutive pregnant women in the second and third trimester of pregnancy registered at our antenatal clinic and satisfying the eligibility criteria were taken in the study after informed consent. Pregnant women with $2-\mathrm{h} \mathrm{PG} \geq 7.8 \mathrm{mmol} / \mathrm{L}$ (DIPSI criterion) were diagnosed as GDM and rest were classified as normal glucose tolerant (NGT) women. One week later all of them were made to undergo the conventional $75 \mathrm{gm}$ OGTT. Data was analyzed using statistical software SPSS ver. 21.

Results: The sensitivity and specificity of DIPSI was $86.8 \%$ and $98.8 \%$ with PPV and NPV of $94.3 \%$ and $97.0 \%$ and overall diagnostic accuracy was $96.5 \%$.

Conclusions: The results of present study show that DIPSI is a simple, single, convenient, economical screening test for GDM and can be used as both diagnostic as well as screening test with good diagnostic efficacy.
\end{abstract}

Keywords: Diabetes, Diagnosis, DIPSI, GDM, OGTT, Pregnancy

\section{INTRODUCTION}

The maternal metabolic adaptation is to maintain the mean fasting plasma glucose of $74.5 \pm 11 \mathrm{mg} / \mathrm{dl}$ and the post prandial peak of $108.7 \pm 16.9 \mathrm{mg} / \mathrm{dl}$. This fine tuning of glycemic level during pregnancy is possible due to the compensatory hyperinsulinemia, as the normal pregnancy is characterized by insulin resistance. A pregnant woman who is not able to increase her insulin secretion to overcome the insulin resistance that occurs even during normal pregnancy develops gestational diabetes. ${ }^{1}$
Gestational Diabetes Mellitus (GDM) is defined as carbohydrate intolerance with recognition or onset during pregnancy, irrespective of the treatment with diet or insulin. The importance of GDM is that two generations are at risk of developing diabetes in the future. Women with a history of GDM are at increased risk of future diabetes, predominately type 2 diabetes, as are their children. ${ }^{1}$

Studies have shown that there is a much higher rate of maternal and fetal compromise in diabetic pregnancies as 
compared with normal pregnancies. ${ }^{2}$ Diabetic mothers are exposed to an increased risk of hypertension in late pregnancy. ${ }^{3}$ Other obstetric complications such as polyhydramnios, preterm labour and abortions are also commonly encountered in pregnant diabetics. Infants of diabetic mothers are exposed to variety of problems such as, sudden intrauterine death, respiratory distress syndrome, hypoglycaemia, cardiomyopathy, neonatal jaundice, impaired calcium and magnesium homeostasis and many more.

A number of studies have documented that the treatment of gestational diabetes as defined by WHO criterion reduced serious perinatal morbidity and also improved the woman's health-related quality of life. ${ }^{4-6}$

American Diabetes Association (ADA) recommends two step procedures for screening and diagnosis of diabetes and that too in selective (high risk) population. ADA recommends 3-hour $100 \mathrm{gm}$ OGTT and Gestational Diabetes Mellitus is diagnosed if any 2 values meet or exceed FPG > $95 \mathrm{mg} / \mathrm{dl}, 1 \mathrm{hr}$ PG > $180 \mathrm{mg} / \mathrm{dl}, 2 \mathrm{hr}$ PG > $155 \mathrm{mg} / \mathrm{dl}$ and $3 \mathrm{hr} \mathrm{PG}>140 \mathrm{mg} / \mathrm{dl} .^{7}$

This procedure requires the pregnant woman to be in a fasting state. It is difficult for the pregnant woman to get up possibly with morning sickness, travel to a clinic and wait an additional two hours before eating. In developing countries such as India, particularly in rural areas, there are other challenges as well to screening for GDM. Some of these challenges include lack of trained phlebotomists, lack of standardized laboratories to do blood glucose estimations, and the problem of transportation.

DIPSI (Diabetes In Pregnancy Study Group India) recommends, a one step procedure with a single glycemic value, to diagnose GDM in the community: It recommends $75 \mathrm{~g}$ OGTT irrespective of fasting status and GDM is diagnosed if 2-hour plasma glucose is $\geq 140 \mathrm{mg} /$ dl. This test correctly identifies subjects with GDM, as well as woman with normal glucose tolerance. ${ }^{8}$ This one step procedure of diagnosing GDM is simple, economical and feasible in Indian scenario.

Hence this prospective study was undertaken to ascertain the validity of DIPSI criterion to diagnose GDM as compared to conventional OGTT and to know the effects of hyperglycemia towards maternal and fetal outcome.

\section{METHODS}

It was a hospital-based screening type of study design conducted during May 2015 to June 2016 at Department of Obstetrics and Gynecology in a tertiary care hospital in Mumbai.

\section{Sampling formulae}

$\mathrm{n}-\mathrm{Z}^{2}$ Sensitivity (1-Sensitivity)/ $\mathrm{L}^{2} * \mathrm{P}$

n-Sample size
$\mathrm{Z}^{2}$-alpha error (at 99\% confidence Interval, value is 2.56 )

$\mathrm{L}^{2}$-allowable error (taken as $5 \%$ of Sensitivity)

P-Prevalence of GDM taken as 20\% [based on present pilot study]

Sensitivity of DIPSI-40\% [Herath et $\mathrm{al}^{5}$ ]

$\mathrm{n}-(2.56)^{2} *(0.4 \times 0.6) /(0.02)^{2} *(0.2)$

n-196

A total of 200 consecutive pregnant women in the second and third trimester of pregnancy registered at antenatal clinic of K J Somaiya Medical College and hospital and satisfying the eligibility criteria were taken in the study after informed consent.

\section{Inclusion criteria}

1. Women with singleton pregnancy.

2. Women aged between $>18$ years.

3 . Women gestational ages ranging between 2428 weeks.

4. Women previously undiagnosed with diabetes, in pre sent pregnancy or previous pregnancy.

\section{Exclusion criteria}

1. Women diagnosed as diabetic in present or previous pregnancy.

2. Women with any comorbid condition such as PIH, T hyroid and Heart conditions.

3. Women with multiple gestation.

\section{Study methodology}

A standardized questionnaire was used and details pertaining to their anthropometrics such as height, weight, BMI, family history, medical history, menstrual history, weeks of gestation (for patients not sure of their dates, the earliest ultrasonography scans were taken into consideration for gestational age), obstetric history, and other relevant information were collected. Their routine obstetric examination was done and after excluding those with multiple gestations or with fetal anomalies by ultrasonography, the subjects were selected according to inclusion and exclusion criteria.

After obtaining the informed consent, pregnant women were given $75 \mathrm{~g}$ oral glucose load irrespective of their last meal timing and venous plasma was drawn at $2 \mathrm{~h}$. The plasma glucose was estimated in the central laboratory by the glucose oxidase peroxidase (GOD-POD) method. Pregnant women with $2-\mathrm{h}$ PG $\geq 7.8 \mathrm{mmol} / \mathrm{L}$ (DIPSI criterion) were diagnosed as GDM and rest were classified as normal glucose tolerant (NGT) women. ${ }^{8}$

One week later all of them were made to undergo the conventional ADA recommended 75 gm OGTT. Authors administered a 75-g anhydrous glucose load after 12 hours fast and obtained fasting, 1-h, and 2-h samples from an antecubital vein. Authors collected samples in tubes containing fluoride and kept them at $4^{\circ} \mathrm{C}$ until 
centrifugation up to $2 \mathrm{~h}$ later. Plasma measurements were performed with glucose oxidase peroxidase (GOD-POD) method. GDM was defined (ADA criteria) as at least two values greater than the following:

Fasting glucose of $>95 \mathrm{mg} \%$

1 -h glucose of $180 \mathrm{mg} \%$, or

2-h glucose of $155 \mathrm{mg} \%$.

\section{Statistical analysis}

All the collected data was entered in Microsoft Excel Sheet 2007.The data was then transferred and analyzed using SPSS ver. 17. Qualitative data was represented in the form of frequency and percentage while quantitative data was represented using Mean \pm S.D. Appropriate statistical evaluation was carried out as per the type and distribution of data. Screening parameters (sensitivity, specificity, etc.) of DIPSI criteria as compared to gold Standard (ADA criteria) was calculated using standard formulae. A p-value of $<0.05$ was taken as level of significance.

\section{RESULTS}

Table 1: Distribution of subjects based on age group.

\begin{tabular}{|lll|}
\hline Age group (years) & $\mathbf{N}$ & $\%$ \\
\hline $20-25$ & 99 & 49.5 \\
\hline $26-30$ & 53 & 26.5 \\
\hline $31-35$ & 37 & 18.5 \\
\hline$>35$ & 11 & 5.5 \\
\hline Total & 200 & 100.0 \\
\hline
\end{tabular}

Almost half of the females were between 20-25 years of age while $5.5 \%$ were over 35 years of age. Although GDM is prevalent among older women, present study had a registering women population mostly in the range of 20-30, hence majority women diagnosed were in the age group of 20-25. The results showing the importance of universal screening for GDM as even younger women now show significant glucose intolerance.

Table 2: Distribution of subjects based on diagnosis of GDM as per OGTT.

\begin{tabular}{|lll|}
\hline Diagnosis (OGTT) & $\mathrm{N}$ & $\%$ \\
\hline GDM & 38 & 19.0 \\
\hline Non GDM & 162 & 81.0 \\
\hline Total & 200 & 100.0 \\
\hline
\end{tabular}

The prevalence GDM as per OGTT was $19 \%$.

Table 3: Distribution of subjects based on diagnosis of GDM as per DIPSI criteria.

\begin{tabular}{|lll|}
\hline Diagnosis (DIPSI) & $\mathbf{N}$ & $\%$ \\
\hline GDM & 35 & 17.5 \\
\hline Non GDM & 165 & 82.5 \\
\hline Total & 200 & 100.0 \\
\hline
\end{tabular}

The prevalence GDM as per DIPSI was $17.5 \%$. Comparison of Table 2 and Table 3 findings show that DIPSI which as a screening test is much easier to perform and more convenient for universal screening, is able to diagnose as many GDM cases (17.5\%) as the more complex and stringent OGTT guidelines (19\%).

Table 4: Comparison of DIPSI and OGTT criteria for diagnosis of GDM.

\begin{tabular}{|c|c|c|c|}
\hline \multirow{2}{*}{ DIPSI } & \multicolumn{2}{|c|}{ OGTT } & \multirow{2}{*}{ Total } \\
\hline & GDM & Non - GDM & \\
\hline GDM & 33 & 2 & 35 \\
\hline Non - GDM & 5 & 160 & 165 \\
\hline Total & 38 & 162 & 200 \\
\hline
\end{tabular}

The sensitivity and specificity of DIPSI was $86.8 \%$ and 98.8\% with PPV and NPV of $94.3 \%$ and $97.0 \%$ and overall diagnostic accuracy was $96.5 \%$.

Table 5: Diagnostic parameters of DIPSI.

\begin{tabular}{|l|l|}
\hline Parameters & $\%$ \\
\hline Sensitivity & 86.8 \\
\hline Specificity & 98.8 \\
\hline PPV & 94.3 \\
\hline NPV & 97.0 \\
\hline Accuracy & 96.5 \\
\hline
\end{tabular}

Considering the sensitivity and specificity of conventional 75 g OGTT as $100 \%$, authors have studied the sensitivity of DIPSI to be $86.8 \%$ as that of OGTT and the specificity of DIPSI to be $98.8 \%$ as that of OGTT which are both comparable. This comparison tells us that DIPSI can confidently replace conventional OGTT as a much easier, simpler to perform one step screening procedure without the fear of under or over diagnosing GDM in the population.

\section{DISCUSSION}

Present hospital-based screening study was conducted with the aim of comparing DIPSI criteria-based test with conventional OGTT for diagnosis of gestational diabetes (GDM) and to compare maternal and perinatal outcome in diabetic and non-diabetic pregnancy.

In present study, the prevalence GDM as per OGTT was $19 \%$ while prevalence as per DIPSI was $17.5 \%$.

Table 6: Comparison of prevalence according to various studies.

\begin{tabular}{|lc|}
\hline Authors & Prevalence of GDM (\%) \\
\hline Seshiah et al & 16.20 \\
\hline Sridhar et al & 12.70 \\
\hline Balaji et al & 13.40 \\
\hline Present study (by OGTT) & 19 \\
\hline Present study (by DIPSI) & 17.5 \\
\hline
\end{tabular}


Depending on the type of population and the diagnostic criteria used, gestational diabetes is said to complicate 1$16 \%$ of all pregnancies. ${ }^{9}$ A random survey performed in India in 2008 in urban population in Chennai showed prevalence of GDM in present country $16.2 \% .^{10}$ Shridhar et al. in a study from Vishakhapatnam observed the prevalence of GDM as $12.7 \% .{ }^{11}$ While when DIPSI recommendation as a diagnostic test was used, prevalence of GDM was $10.2 \%$. In a study by Balaji et al using DIPSI criterion $13.4 \%$ of women were identified as GDM. ${ }^{12}$ The recent data on the prevalence of GDM in present country was $16.55 \%$ by WHO criteria of 2 hours $\mathrm{PG} \geq 140 \mathrm{mg} / \mathrm{dl}^{2}$

\section{Diagnostic accuracy}

In present study, sensitivity and specificity of DIPSI was $86.8 \%$ and $98.8 \%$ with PPV and NPV of $94.3 \%$ and $97.0 \%$ and overall diagnostic accuracy was $96.5 \%$. The study showed almost all women diagnosed as GDM by $75 \mathrm{~g}$ glucose non-fasting test also satisfied the diagnostic criteria of 75-g oral glucose test performed in the fasting state recommended by WHO.

In a recent study, Seshiah et al done on pregnant women with no previous history of GDM/ pre GDM showed no significant difference in diagnosing GDM by the two criteria -by DIPSI criterion, the prevalence was $13.4 \%$, applying IADPSG recommendation the prevalence of GDM was $14.6 \%$ and concluded that there was little difference in the diagnostic accuracy of the two tests. ${ }^{13}$ Thus DIPSI method is a suitable test for screening and diagnosing GDM in Indian population.

Table 7: Comparison of studies for sensitivity and specificity.

\begin{tabular}{|lll|}
\hline Authors & \multicolumn{2}{c|}{ DIPSI } \\
\hline Sharma A et al & $90.20 \%$ & Specificity \\
\hline Polur et al & $82.50 \%$ & $97.50 \%$ \\
\hline Present study & $86.80 \%$ & $93.00 \%$ \\
\hline
\end{tabular}

Similar results were also observed by Sharma et al. where sensitivity and specificity of DIPSI was observed as $90.2 \%$ and $97.5 \%$ respectively. ${ }^{14}$ Polur et al observed a sensitivity and specificity of DIPSI as $82.5 \%$ and $93 \%$ respectively. ${ }^{15}$ Balaji $\mathrm{V}$ et al in their study concluded that DIPSI criterion is cost-effective and evidence-based procedure meets present responsibility of offering a single-step definitive glucose test to every pregnant woman belonging to any socio-economic status.

\section{CONCLUSION}

Gestational diabetes mellitus is highly prevalent in mothers attending present antenatal clinics. The results of present study show that DIPSI is a simple, single, convenient, economical screening test for GDM and can be used as both screening as well as diagnostic test with good diagnostic efficacy. So, it can replace OGTT as gold standard and can be used in routine practice to diagnose GDM.

Also, as gestational diabetes mellitus is associated with myriad of adverse maternal and fetal outcomes like hypertensive disorders in pregnancy, vaginal candidiasis, post-datism, polyhydramnios, high birth weight, shoulder dystocia and hypoglycemia in neonates and still birth. Thus, routine screening for Gestational Diabetes and its associated complications is paramount to reduce GDM related morbidity and mortality among mothers and the neonates.

Funding: No funding sources

Conflict of interest: None declared

Ethical approval: The study was approved by the Institutional Ethics Committee

\section{REFERENCES}

1. Yogev Y, Chen R, Langer O, Hod M. Diurnal Glycemic profile characterization in non-diabetic non-obese subjects during the first trimester. The 37th Annual Meeting of the Diabetes and Pregnancy Study Group, Myconos-Hellas: September 2005.

2. American Diabetes Association; Gestational diabetes mellitus (Position statement). Diabetes Care. 2004;27(Suppl 1):S88-90.

3. Kjos SL, Buchanan TA. Gestational diabetes mellitus. New Eng J Med. 1999;341(23):1749-56.

4. Crowther CA, Hiller JE, Moss JR, McPhee AJ, Jeffries WS, Robinson JS, et al. Effect of treatment of gestational diabetes mellitus. N Engl J Med. 2005; 352:2477-86.

5. Gayle C, Germain S, Marsh MS, Rajasingham D, Brackenridge A, Carroll $\mathrm{P}$, et al. Comparing pregnancy outcomes for intensive versus routine antenatal treatment of GDM based on a $75 \mathrm{gm}$ OGTT 2- $\mathrm{h}$ blood glucose (>140 $\mathrm{mg} / \mathrm{dl})$. Diabetologia. 2010;53:S435.

6. Wahi P, Dogra V, Jandial K, Bhagat R, Gupta R, Gupta S, et al. Prevalence of Gestational Diabetes Mellitus (GDM) and its outcomes in Jammu region. J Assoc Physicians India. 2011;59:227-30.

7. Cosson E. Screening and insulin sensitivity in gestational diabetes. Abstract volume of the 40th Annual Meeting of the EASD, September 2004: A 350.

8. V Seshiah. DIPSI Guidelines - Kolkata declaration, fifth national conference of diabetes in pregnancy study group, India. The $\mathrm{J}$ Assoc Phys India. 2010;58:329-30.

9. JimennezMoLeon J, Bueno-Cavanillas A, Luna-DelCastillo J D, Lardelli-Claret P, García-Martin M, Gálvez-Vargas R. Predictive value of screen for GDM, Influence of associated risk factors. Acta Obstet Gynaecol. 2000;79:991-8.

10. Seshiah V, Balaji V, Balaji MS, Paneerselvam A, Arthi T, Thamizharasi M, Datta M. Prevalence of 
gestational diabetes mellitus in South India (Tamil Nadu): a community-based study. JAPI. 2008 May;56:329-3.

11. Sridhar GR, Nagmani G. Gestational diabetes and other endocrine disorders during pregnancy. J Obstet Gynaecol India. 2003;35:140.

12. Balaji V, Balaji M, Anjalakshi C, Cynthia A, Arthi T, Seshiah V. Diagnosis of gestational diabetes mellitus in Asian-Indian women. Indian J Endocrinol Metabol. 2011 Jul 1;15(3):187.

13. Seshiah V, Balaji V, Balaji MS, Sanjeevi CB, Green A. Gestational diabetes mellitus in India. J Assoc Phys India. 2004;52:707-11.
14. Sharma A, Gupta M, Agrawal A. Comparison of diagnostic accuracy of two one step procedures for screening of gestational diabetes mellitus. Int $\mathrm{J}$ Reprod Contracept Obstet Gynecol. 2015;4(1):81-5.

15. Polur PK, Bandela PV, Hindumathi SS. Diabetes in pregnancy study group in India (DIPSI): a novel criterion to diagnose. GDM. 2016;10:1-6.

Cite this article as: Desai GG, Sonawane P. Comparison of DIPSI guidelines versus conventional OGTT for diagnosis of gestational diabetes mellitus. Int J Reprod Contracept Obstet Gynecol 2018;7:3168-72. 